\title{
Polyhedral meshing in numerical analysis of conjugate heat transfer
}

\author{
Marcin Sosnowski ${ }^{1, *}$, Jaroslaw Krzywanski ${ }^{1}$, Karolina Grabowska ${ }^{1}$, and Renata Gnatowska ${ }^{2}$ \\ ${ }^{1}$ Jan Dlugosz University in Czestochowa, Faculty of Mathematics and Natural Sciences, 13/15 Armii Krajowej Av, 42-200 \\ Czestochowa, Poland \\ ${ }^{2}$ Czestochowa University of Technology, Faculty of Mechanical Engineering and Computer Science, 21 Armii Krajowej Av, $42-200$ \\ Czestochowa, Poland
}

\begin{abstract}
Computational methods have been widely applied in conjugate heat transfer analysis. The very first and crucial step in such research is the meshing process which consists in dividing the analysed geometry into numerous small control volumes (cells). In Computational Fluid Dynamics (CFD) applications it is desirable to use the hexahedral cells as the resulting mesh is characterized by low numerical diffusion. Unfortunately generating such mesh can be a very time-consuming task and in case of complicated geometry - it may not be possible to generate cells of good quality. Therefore tetrahedral cells have been implemented into commercial pre-processors. Their advantage is the ease of its generation even in case of very complex geometry. On the other hand tetrahedrons cannot be stretched excessively without decreasing the mesh quality factor, so significantly larger number of cells has to be used in comparison to hexahedral mesh in order to achieve a reasonable accuracy. Moreover the numerical diffusion of tetrahedral elements is significantly higher. Therefore the polyhedral cells are proposed within the paper in order to combine the advantages of hexahedrons (low numerical diffusion resulting in accurate solution) and tetrahedrons (rapid semi-automatic generation) as well as to overcome the disadvantages of both the above mentioned mesh types. The major benefit of polyhedral mesh is that each individual cell has many neighbours, so gradients can be well approximated. Polyhedrons are also less sensitive to stretching than tetrahedrons which results in better mesh quality leading to improved numerical stability of the model. In addition, numerical diffusion is reduced due to mass exchange over numerous faces. This leads to a more accurate solution achieved with a lower cell count. Therefore detailed comparison of numerical modelling results concerning conjugate heat transfer using tetrahedral and polyhedral meshes is presented in the paper.
\end{abstract}

\section{Introduction}

The application of Computational Fluid Dynamics (CFD) as an advanced and valuable research tool has recently been common in various branches of industry such as aerospace [1], automotive [2,3], civil engineering [4-8], power engineering [9], medicine [10] and others. It allows to achieve flawless product designing at relatively low cost by combining the prediction of fluid flow, heat and mass transfer, chemical reactions and related phenomena.

CFD is a numerical approach for solving nonlinear differential equations describing the analysed fluid flow. Majority of these equations do not have analytical solution hence approximate numerical methods are applied in order to solve them. The level of approximation directly influences the compatibility of modelling results with real data. The round-off errors due to finite word size will always interfere the solution but the truncation errors due to approximations in numerical models can be minimized by improved pre-processing (meshing), which is the first step in CFD analysis. This stage of CFD consists in discretizing the computational domain into numerous finite volumes (cells). Refining the computational mesh strongly contributes to the numerical stability of the model and consequently the accuracy of the obtained results $[11,12]$ because it drastically reduces the truncation error. Moreover the type of mesh elements strongly influences the numerical diffusion as well as the quality and time of convergence. Two mesh types are commonly applied in CFD: hexahedral HEX elements (Fig. 1 red) and tetrahedral TETRA elements (Fig. 1 blue)

HEX mesh is characterized by low numerical diffusion, hence in most cases it results in the lowest discretization error. But on the other hand the numerical diffusion increases in case of flow not perpendicular to the cell faces and it is not always possible to construct structured HEX mesh for complex geometries. Therefore this mesh type is frequently ineffective in industrial applications.

For that reason algorithms of generation TETRA mesh have been implemented into commercial pre-processors. The generation of this type of mesh can be automated at a large degree, which is the biggest advantage of TETRA mesh. But such cells cannot be stretched excessively, so

\footnotetext{
"Corresponding author: m.sosnowski@ajd.czest.pl
} 
significantly larger number of elements has to be used in comparison to structured HEX mesh in order to represent specific geometry issues. Moreover the neighbouring nodes of a TETRA cell may lie in nearly one plane and therefore computing gradients can be difficult, which may result in poor convergence [10].

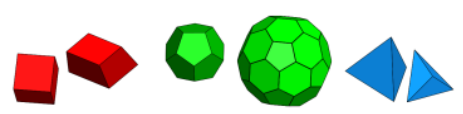

Fig. 1. Types of mesh elements:

HEX - red, TETRA - blue, POLY - green.

POLY mesh has recently been introduced in order to combine the advantages of HEX and TETRA meshes: low numerical diffusion of HEX and rapid semi-automatic generation of TETRA $[5,13,14]$. The individual POLY neighbours with more cells in comparison to HEX and TETRA, which improves the calculation of gradients and allows to interchange the mass over numerous faces, reducing numerical diffusion effects caused by flows not perpendicular to any of the cell face. It is advantageous in situations where no prevailing flow direction can be identified and leads to more accurate solution achieved with a lower cell count. Moreover POLY cell is not oversensitive to stretching and therefore assures improved numerical stability of the model.

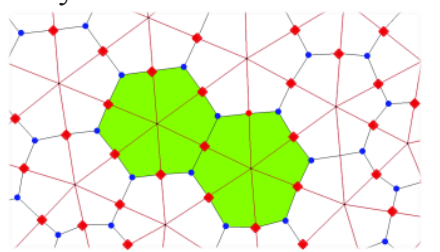

Fig. 2. Conversion of TETRA mesh to POLY mesh

POLY mesh generation is a simple conversion of TETRA mesh to POLY mesh by decomposition of cell into multiple sub-volumes depicted in Fig. 2. In order to do so, new edges are created on each face between the face centroid (dots in Fig. 2) and the centroids of the edges of that face (hexagons in Fig. 2). Subsequently new faces are created within the cell by connecting the cell centroid to the new edges on each face. The newly-created faces may be adjusted and merged with the neighbouring ones during the agglomeration process in order to minimize the number of faces of the resultant polyhedral cell.

\section{Methods}

\subsection{The research object}

Air-water heat exchanger was the research object. It consisted of the insulated cuboid $(180 \mathrm{~mm} \times 120 \mathrm{~mm} \times$ $60 \mathrm{~mm}$ ) with air inlet/outlet and aluminium heat pipe of $8 \mathrm{~mm}$ internal radius and $1 \mathrm{~mm}$ wall thickness (Fig. 3). The CAD model of the heat exchanger was prepared in SolidWorks as an assembly of three parts: cuboid-shaped air domain, aluminium pipe and domain of water in the heat pipe. ANSYS 18.1 plugin was used in order to parametrize the CAD model for subsequent meshing and analysis by defining the named selections for boundary conditions assignment. The plugin allows also the bi-directional associativity between the CAD geometry and mesh generator. Moreover the model was split into two symmetric parts in order to minimize the volume of the computational domain which directly influences the CPU time.
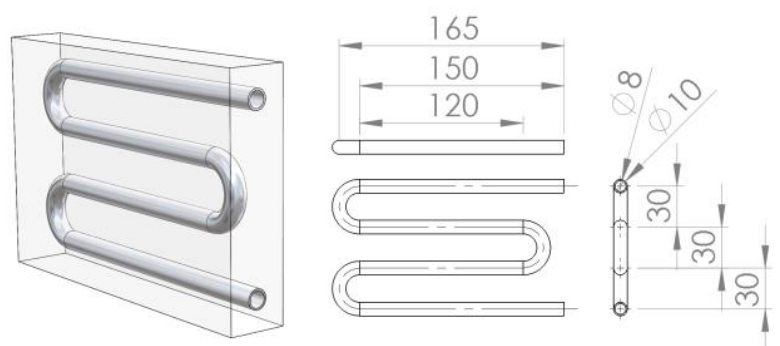

Fig. 3. The analysed heat exchanger

\subsection{Pre-processing}

ANSYS Meshing 18.1 was used as a pre-processor. The patch conforming algorithm was applied in order to generate fully conformal tetrahedral mesh. Element size representing the aluminium heat pipe as well as maximal face and TETRA sizes allowed in the computational domain were selected as mesh size constrains. Four configurations for both mesh types depicted in Fig. 4 (TETRA and POLY) were generated in order to perform mesh dependency studies. The mesh configurations differed in the total number of elements but the relative mesh sizes were maintained. The POLY meshes were generated using ANSYS Fluent 18.1 by conversion of the TETRA mesh. The number of elements of the final meshes of both analysed types was approx. 60k, 150k, $230 \mathrm{k}$ and $340 \mathrm{k}$.
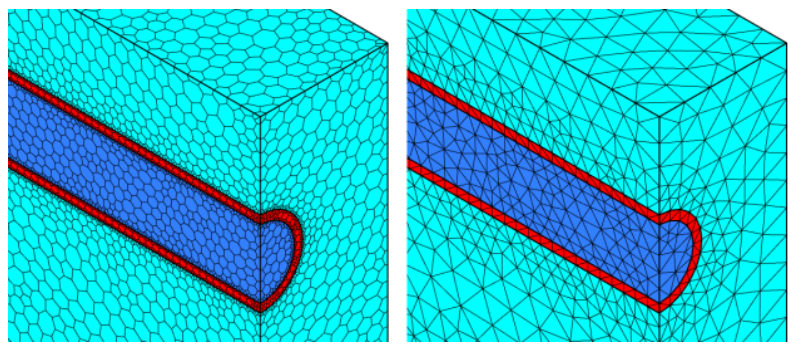

Fig. 4. Exemplary POLY (left) and TETRA (right) meshes

The output mesh quality analysis provided a composite metric that ranges between 0 and 1 according to formula (1). A value of 1 indicates a perfect element while a value of 0 indicates that the element has a zero or negative volume. The element of the worst quality generated throughout the entire research (eight meshes) was characterized by the quality index of 0.11 and therefore it was assumed that the mesh did not affect the numerical stability of the computational model.

$$
Q=C \cdot \frac{V}{\sqrt{\left(\sum l^{2}\right)^{3}}}
$$

where :

$V \quad$ volume of the computational domain;

$l \quad$ length of the element edge;

$C$ constant dependent on the element type. 


\subsection{Numerical accuracy and discretization error}

The numerical accuracy and its estimation is crucial for CFD. One issue connected with the numerical accuracy estimation is the iterative convergence. In most cases it can be assumed that it is achieved with at least three orders of magnitude decrease in the normalized residuals for each equation solved. This guideline was applied within the carried out analysis.

The mesh resolution is also a crucial factor influencing the numerical accuracy [15]. One of the methods of discretization error estimation is the Richardson Extrapolation (RE) [16]. The local RE values of the predicted variables may not exhibit a monotonic dependence on mesh resolution. Moreover in transient calculations, RE will also be a function of time and space. Despite its shortcomings and generalizations, it is currently the most reliable method available for the prediction of numerical uncertainty.

The mesh dependency analysis applied within this paper to the estimation of discretization error is based on the RE. The representative mesh size $h$ was defined according to equation (2) [16].

$$
h=\sqrt[3]{\frac{1}{N} \sum_{i=1}^{N}\left(\Delta V_{i}\right)}
$$

where:

$\Delta V_{i}$ - the volume of the $\mathrm{i}^{\text {th }}$ cell,

$N$ - the total number of cells.

The mesh refinement factor $r$ was calculated as quotient of representative size of coarse and fine mesh (3) with assumption (4).

$$
\begin{gathered}
r=\frac{h_{\text {coarse }}}{h_{\text {fine }}} \\
h_{1}<h_{2}<h_{3} ; \quad r_{21}=\frac{h_{2}}{h_{1}} ; r_{32}=\frac{h_{3}}{h_{2}}
\end{gathered}
$$

The order of convergence $p$ was calculated based on equation (5) where $\varepsilon_{32}=\phi_{3}-\phi_{2}$ and $\varepsilon_{21}=\phi_{2}-\phi_{1}$. It was solved using fixed-point iteration with the initial guess equal to the first term.

$$
p=\frac{|\ln | \frac{\varepsilon_{32}}{\varepsilon_{21}}\left|+\ln \left(\frac{r_{21}^{p}-1 \cdot \operatorname{sgn}\left(\frac{\varepsilon_{32}}{\varepsilon_{21}}\right)}{r_{32}^{p}-1 \cdot \operatorname{sgn}\left(\frac{\varepsilon_{32}}{\varepsilon_{21}}\right)}\right)\right|}{\ln \left(r_{21}\right)}
$$

$\phi_{k}$ denotes the value of the variable important to the objective of the simulation study for solution obtained with the $k^{\text {th }}$ mesh. The average temperature on outlet of the domain was chosen as the most representative variable.

The extrapolated values were calculated on the basis of equation (6), approximate relative error on the basis of eq. (7), extrapolated relative error on the basis of eq. (8) and mesh convergence index $C$ on the basis of eq. (9).

$$
\phi_{\text {ext }}^{21}=\frac{r_{21}^{p} \phi_{1}-\phi_{2}}{r_{21}^{p}-1} ; \quad \phi_{\text {ext }}^{32}=\frac{r_{32}^{p} \phi_{2}-\phi_{1}}{r_{32}^{p}-1}
$$

$$
\begin{gathered}
e_{a}^{21}=\left|\frac{\phi_{1}-\phi_{2}}{\phi_{1}}\right| ; \quad e_{a}^{32}=\left|\frac{\phi_{2}-\phi_{3}}{\phi_{2}}\right| \\
e_{\text {ext }}^{21}=\left|\frac{\phi_{\text {ext }}^{12}-\phi_{1}}{\phi_{\text {ext }}^{12}}\right| ; \quad e_{\text {ext }}^{32}=\left|\frac{\phi_{\text {ext }}^{23}-\phi_{2}}{\phi_{\text {ext }}^{23}}\right| \\
C_{21}=\frac{1.25 \cdot e_{a}^{21}}{r_{21}^{p}-1} ; \quad C_{32}=\frac{1.25 \cdot e_{a}^{32}}{r_{32}^{p}-1}
\end{gathered}
$$

The type of convergence can be evaluated using formula (10).

$$
\frac{\varepsilon_{\text {coarse }}}{\varepsilon_{\text {fine }}}\left\{\begin{array}{lr}
<0 & \text { converged (oscilatory) } \\
>1 & \text { not converged } \\
{[0,1]} & \text { converged }
\end{array}\right.
$$

\begin{tabular}{|c|c|c|c|c|c|c|}
\hline & 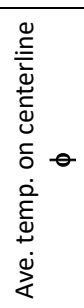 & 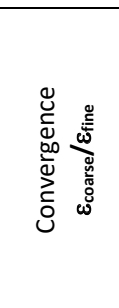 & 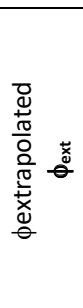 & 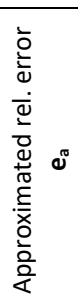 & 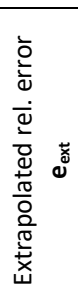 & 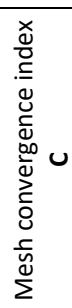 \\
\hline POLY_340k & 312,73 & converged & 312,73 & $0,01 \%$ & $0,00 \%$ & $0,00 \%$ \\
\hline POLY_230k & 312,76 & not conv. & 312,76 & $0,00 \%$ & $0,00 \%$ & $0,00 \%$ \\
\hline POLY_150k & 312,77 & - & - & $0,03 \%$ & - & - \\
\hline POLY_60k & 312,87 & - & & & & \\
\hline TETRA_340k & 312,76 & converged & 312,71 & $0,02 \%$ & $0,02 \%$ & $0,02 \%$ \\
\hline TETRA_230k & 312,82 & osc. conv. & 312,63 & $0,02 \%$ & $0,06 \%$ & $0,01 \%$ \\
\hline TETRA_150k & 312,88 & - & & $0,03 \%$ & - & - \\
\hline TETRA_60k & 312,79 & - & & & & \\
\hline
\end{tabular}

In order to evaluate the discretization error, four TETRA and four POLY meshes were generated of approx. 60k, 150k, 230k and 340k cells. As can be seen in Table 1, the meshes of $340 \mathrm{k}$ cells can be assumed as converged.

Table 1. Discretization errors.

\subsection{Computational model}

The computational models used for all eight cases (four configurations of TETRA mesh and four configurations of POLY mesh) were parametrized identically. The only difference was the computational mesh. The solver was configured as pressure-based and the analysis were performed for steady state. Standard k-epsilon viscous model was used along with standard wall functions. The turbulence model constants are listed in Table 2.

Table 2. Turbulence model parameters.

\begin{tabular}{|c|c|}
\hline Model Constant & Value \\
\hline Cmu & 0.09 \\
\hline C1-Epsilon & 1.44 \\
\hline C2-Epsilon & 1.92 \\
\hline TKE Prandtl Number & 1 \\
\hline TDR Prandtl Number & 1.3 \\
\hline
\end{tabular}

Velocity-inlet boundary condition type was assigned to the inlet to the air domain with velocity magnitude normal to the boundary equal $0.1 \mathrm{~m} / \mathrm{s}$ (Fig. 5). The turbulence parameters were specified as intensity and hydraulic diameter equal $5 \%$ and $0.09 \mathrm{~m}$ respectively. The temperature of the inlet air was $293 \mathrm{~K}$. The water inlet was 
also configured as velocity-inlet with velocity magnitude normal to the boundary equal $0.01 \mathrm{~m} / \mathrm{s}$. The turbulence parameters were specified as intensity and hydraulic diameter equal $5 \%$ and $0.008 \mathrm{~m}$ respectively. The temperature of the inlet air was $323 \mathrm{~K}$.

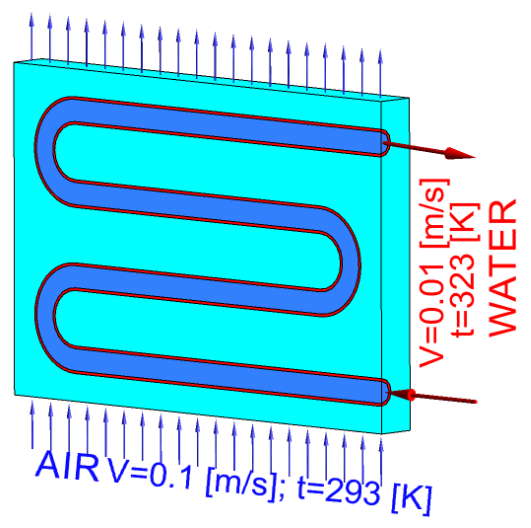

Fig. 5. Boundary conditions

Two fluids (liquid-water, air) and one solid (aluminium) of parameters given in Table 3 were chosen as cell zone conditions.

The outlet of the computational domain was defined as pressure outlet. Symmetry boundary condition was defined on the symmetry plane. The air-aluminium and water-aluminium interface surfaces were defined as thermally coupled in order to assure the conjugate heat transfer. Wall type condition was assigned to the remaining surfaces.

Table 3. Parameters of cell zones.

\begin{tabular}{|c|c|c|c|c|}
\hline material & 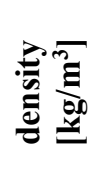 & 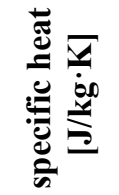 & 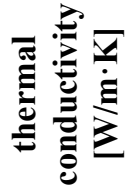 & 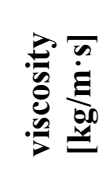 \\
\hline water-liquid & 998.2 & 4182 & 0.6 & $1003 \mathrm{e}-6$ \\
\hline air & 1.225 & 1006,43 & 0,0242 & $17.89 \mathrm{e}-6$ \\
\hline aluminium & 2719 & 871 & 202.4 & - \\
\hline
\end{tabular}

Pressure-velocity coupling by SIMPLE algorithm was used as a solution method. This algorithm uses a relationship between velocity and pressure corrections to enforce mass conservation and obtain the pressure field.

\section{Results}

\subsection{Convergence}

The numerical calculations were performed with ANSYS Fluent 18.1 on Windows 10 workstation equipped with Intel i7 4510U 2.00GHz CPU and 16GB of RAM. The solver was operating in a single mode.

The analysis were assumed as converged when all normalized residuals decreased below the level of 1e-3.

The number of iterations to convergence varied dependent on the mesh element type and the number of mesh elements as depicted in Fig. 6. The converged solution was obtained with fewer iterations in case of POLY mesh regardless of the number of mesh elements. The reduction of iterations varied between $20 \%$ (150k cells) and $32 \%$ (340k cells).

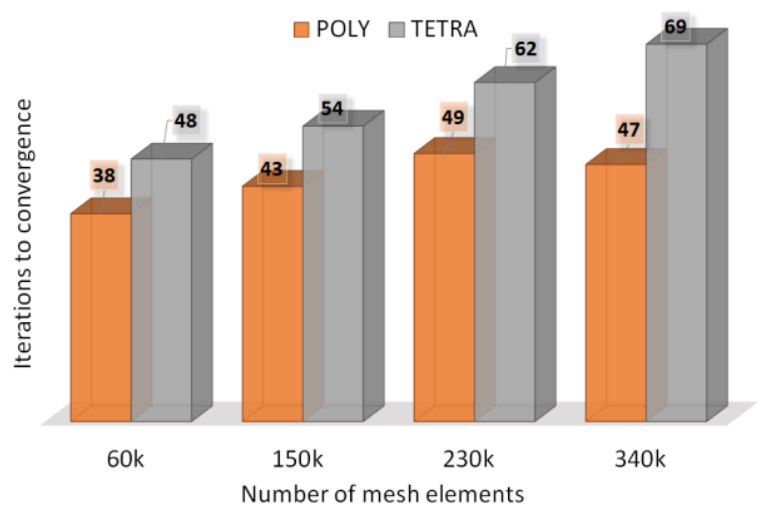

Fig. 6. Number of iterations to convergence of the analysed cases

Moreover the calculation time to convergence was measured with a stopwatch in order to evaluate the CPU time necessary to obtain the solution. The comparison of the calculation time for all analysed meshes is depicted in Fig. 7. The reduction of calculation time for POLY mesh was observed in case of the $340 \mathrm{k}$ cells ( $18 \%$ reduction) while calculations with coarser meshes were approx. $15 \%$ slower in comparison to TETRA.

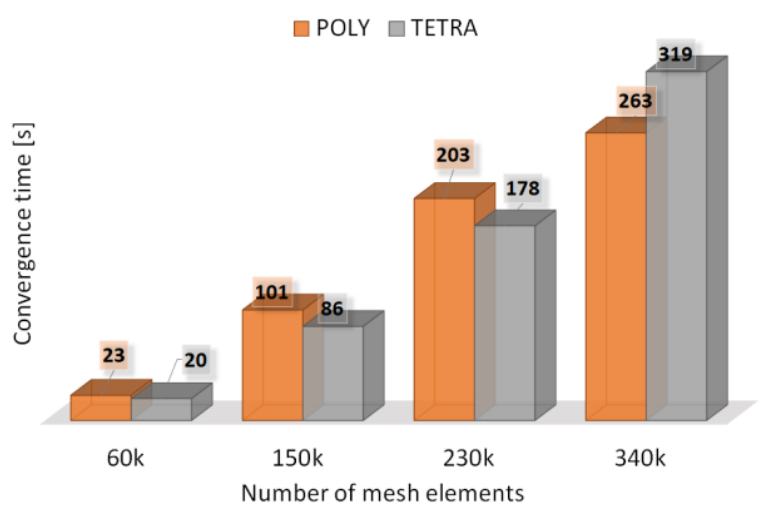

Fig. 7. Time to convergence of the analysed cases

\subsection{Temperature field}

The temperature distribution in the computational domain and in the air outlet from the heat exchanger is depicted for both mesh types and all mesh resolutions in Fig. 8 and Fig. 9. The figures are created with exactly the same temperature scale in order to easily distinguish the differences in the temperature fields resulting from the mesh type as well as the number of mesh elements.

Both figures reveal evident influence of the number of mesh elements on the results in case of TETRA while the results for POLYs are almost identical except for the mesh of the lowest element count. Moreover the temperature field for TETRAs is characterised by the artefacts in the form of jagged edges of the colour bands which directly indicates the discretization error. 

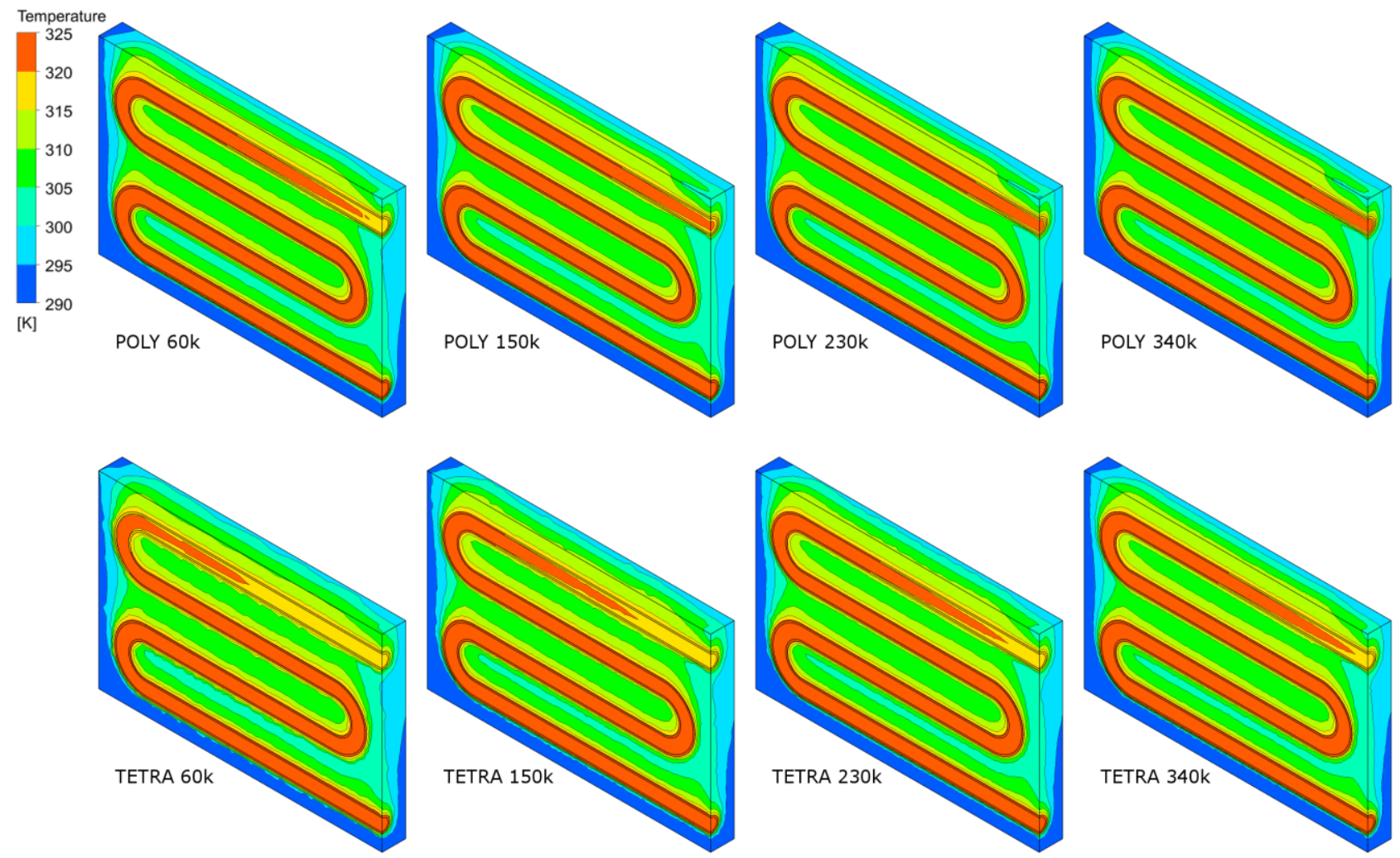

Fig. 8. Temperature distribution in the computational domain
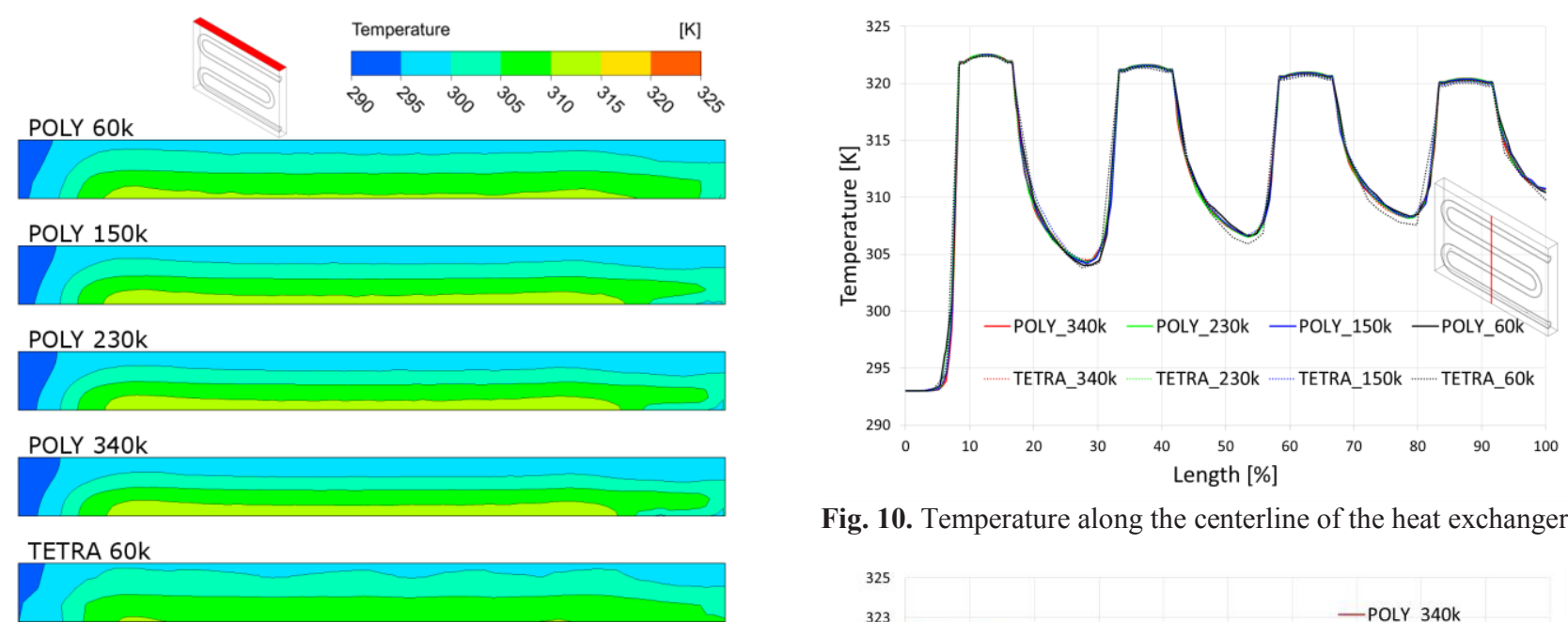

Fig. 10. Temperature along the centerline of the heat exchanger
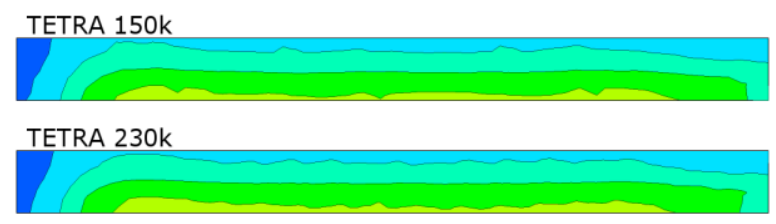

TETRA 340k

Fig. 9. Temperature distribution in the air outlet surface

\subsection{Temperature profile}

The variation of air temperature along the centerline (Fig. 10) of the analysed heat exchanger depicts the efficiency of conjugate heat transfer between the fluid/aluminium/air domains.

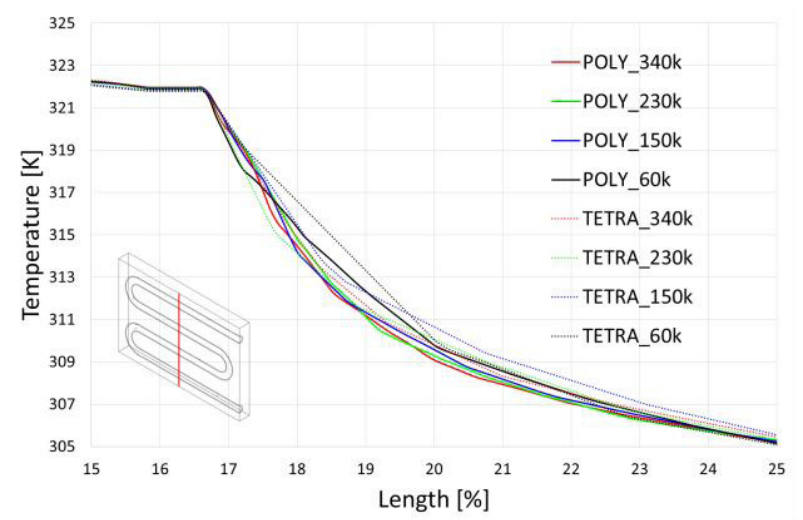

Fig. 11. Temperature along the segment of the centerline of the heat exchanger

The temperature profile obtained with all POLY meshes is similar but the results for TETRA mesh of $60 \mathrm{k}$ 
elements is noticeably different. The segment of the centerline concerning the $15 \%-25 \%$ of the heat exchanger length depicted in Fig. 11 allows to clearly visualize the difference in the efficiency of conjugate heat transfer due to mesh type and number of mesh elements. It confirms the rough estimation that the dispersion between the TETRA meshes of different number of elements is significantly greater in comparison to POLY meshes.

\section{Discussion}

The presented results of conjugate heat transfer analysis with the use of computational methods proves the importance of the very first step in CFD analysis which is the pre-processing. The obtained temperature field and temperature profile differed not only due to mesh resolution (number of cells) but also due to the mesh type (TETRA/POLY).

The carried out research confirmed that the discretization of the computational domain with POLY elements is beneficial not only in terms of the number of iterations necessary to obtain the converged solution but also the homogeneity of the results obtained with different mesh resolutions. Therefore the performed research prove that the application of POLY mesh leads to a more accurate solution achieved with a lower cell count.

Experimental validation of the numerical results is necessary in order to properly and fully define the applicability of polyhedral meshing in conjugate heat transfer numerical analysis.

\section{Conclusions}

The carried out research indicate the purposefulness of further investigation concerning the application of polyhedral elements in CFD.

Detailed experimental research is necessary in order to validate the computational results against real data. The experimental test stand allowing to perform the above mentioned experimental tests is under construction.

The work is partialy suported by Narodowe Centrum Nauki (National Science Centre) grant number 2017/01/X/ST8/00019

\section{References}

1. Kroll, N., The ADIGMA Project, in Adigma-a European Initiative on the Development of Adaptive Higher-Order Variational Methods for Aerospace Applications: Results of a Collaborative Research Project Funded by the European Union, 2006-2009, N. Kroll, et al., Editors. 2010, Springer-Verlag Berlin: Berlin. p. 1-9.

2. Jamrozik, A., et al., Numerical simulation of two-stage combustion in SI engine with prechamber. Applied Mathematical Modelling, 2013. 37(5): p. 2961-2982.

3. Jamrozik, A., et al., Modeling of Thermal Cycle CI Engine with Multi-Stage Fuel Injection. Advances in Science and Technology Research Journal, 2017. 37(5): p. 179-186.
4. Gnatowska, R., A Study of Downwash Effects on Flow and Dispersion Processes around Buildings in Tandem Arrangement. Polish Journal of Environmental Studies, 2015. 24(4): p. 1571-1577.

5. Lanzafame, R., S. Mauro, and M. Messina, Wind turbine CFD modeling using a correlation-based transitional model. Renewable Energy, 2013. 52: p. 31-39.

6. Gnatowska, R., M. Sosnowski, and V. Uruba, CFD modelling and PIV experimental validation of flow fields in urban environments. E3S Web of Conferences, 2017. 14.

7. Gnatowska, R. and T. Rybak, Numerical Analysis of Heat Transfer around 2D Circular Cylinder in Pulsation Inflow, in Proceedings of the International Conference of Numerical Analysis and Applied Mathematics 2014, T.E. Simos and C. Tsitouras, Editors. 2015.

8. Gnatowska, R. and S. Sikora, Numerical Analysis of Wind Flow and Erosion in Flow around the Bump Terrain, in Proceedings of the International Conference of Numerical Analysis and Applied Mathematics 2014, T.E. Simos and C. Tsitouras, Editors. 2015.

9. Zylka, A., et al. Numerical simulations of fluidization dynamics in a hot model of a CLC process. in 4th Scientific and Technical Conference on Modern Technologies and Energy Systems (WTiUE). 2016. Cracow, POLAND.

10. Spiegel, M., et al., Tetrahedral vs. polyhedral mesh size evaluation on flow velocity and wall shear stress for cerebral hemodynamic simulation. Computer Methods in Biomechanics and Biomedical Engineering, 2011. 14(1): p. 9-22.

11. Bianchi, G., et al., Deforming grid generation for numerical simulations of fluid dynamics in sliding vane rotary machines. Advances in Engineering Software, 2017. 112: p. 180-191.

12. Li, H., L. Rong, and G.Q. Zhang, Reliability of turbulence models and mesh types for CFD simulations of a mechanically ventilated pig house containing animals. Biosystems Engineering, 2017. 161: p. 37-52.

13. Su, J.W., et al., A two-layer mesh method for discrete element simulation of gas-particle systems with arbitrarily polyhedral mesh. International Journal for Numerical Methods in Engineering, 2015. 103(10): p. 759-780.

14. Sosnowski, M., J. Krzywanski, and R. Gnatowska, Polyhedral meshing as an innovative approach to computational domain discretization of a cyclone in a fluidized bed CLC unit. E3S Web of Conferences, 2017. 14: p. 01027.

15. Eca, L. and M. Hoekstra, A procedure for the estimation of the numerical uncertainty of CFD calculations based on grid refinement studies. Journal of Computational Physics, 2014. 262: p. 104-130.

16. Celik, I.B., et al., Procedure for estimation and reporting of uncertainty due to discretization in CFD applications. Journal of Fluids EngineeringTransactions of the Asme, 2008. 130(7). 